Sawyer, Helen B. Variable stars in the globular cluster Messier 9.

A blink survey of 30 pairs of plates by the writer, followed by measurement of 78 plates from the Steward and David Dunlap Observatories, now adds the relatively bright globular cluster Messier 9 (NGC 6333) to the list of clusters in which variables have been studied. Messier 9 is among the conspicuous clusters, but because of its declination of $-18^{\circ}$ it is not easily studied from northern latitudes.

One variable star was discovered by Shapley in I9I6. Fourteen variables, including this one, were found in the recent survey. Measures on 78 plates taken over an 8-year interval indicate that the variables are of the RR Lyrae type; there appears to be no bright long-period Cepheid associated with this cluster. The period determination is not yet complete, but two groups of periods appear to be represented. Four periods have been determined between 0.57 and 0.60 day; two stars have periods of 0.38 and 0.28 day. There is a suggestion that the longer period variables average about o.I 5 mag. brighter than the shorter period stars. Several of the variables are among the brightest stars at maximum, reaching $15.6 \mathrm{mag}$. The average magnitude of the 25 brightest stars as determined from the same sequence is 15.50 . The mean median magnitude for ten cluster type variables is $\mathbf{I} 6.37$, which may be taken as the modulus of the cluster. The color-excess as given by Stebbins and Whitford is +0.24 , so the total photographic absorption is probably 2 magnitudes. This suggests a distance of about seven and a half kiloparsecs for this cluster.

The number of clusters examined for variables is increasing very slowly now, as more difficult objects are being studied, but the number of variables being found is still increasing substantially. In I930, 45 clusters had been examined, and 886 variables found; by I 939,60 clusters had been searched and I I 6 variables found. Now, in 1947, 62 clusters have been examined and I307 variables discovered.

David Dunlap Observatory,
University of Toronto,
Richmond Hill, Ontario.

\section{Seyfert, Carl K. Five thousand external galaxies and a new dense group.}

Nine thirty-minute photographic plates taken by Dr. F. D. Miller with the $24-33$ inch Harvard Schmidt telescope (J series) were made available for this investigation through the kindness of Dr. Harlow Shapley. For comparison purposes one three-hour plate taken with the I6-inch Metcalf telescope (MC series) was also used. This latter plate, typical of the plates used at Harvard in the survey of the northern galaxies to the I 7 th magnitude, partially overlaps two of the Schmidt plates. The regions studied lie in middle galactic latitudes, close to $\mathrm{o}^{\circ}$ and $\mathrm{I} 80^{\circ}$ galactic longitudes, in Leo, Serpens, and Hercules.

A search of the plates at the Barnard Observatory of Vanderbilt University revealed approximately 5000 new galaxies and photographic magnitudes were obtained for 3500 of these. On the average the magnitude limit for stars was I7.6 and the magnitude of the maximum frequency of galaxies on each plate was found to be 0.3 mag. brighter than the stellar limit. The survey in these regions is believed to be complete to at least $17.0 \mathrm{mag}$.

For the 555 galaxies common to the $\mathrm{J}$ and MC plates the magnitudes from the Schmidt plates were found to be o.I6 mag. fainter than those found from the Metcalf plate. The stellar images on the $\mathrm{J}$ plates were considerably smaller than those on the MC plate and the nebular images more distinct. For this reason, the nebular magnitudes determined from the MC plate are probably superior to those determined from the Schmidt plates. However, the discovery of galaxies is more certain on the Schmidt plates and the limit of completeness for galaxies found on Schmidt plates was found to be nearer the stellar limit than that for the Metcalf telescope.

A plot of $\log N_{m}$ against $m$, corrected for red shift as obtained from Hubble's paper, ${ }^{1}$ for each of the plates showed that the galaxies follow fairly closely the straight line representing uniform distribution in depth:

$$
\log N_{m}=0.6 m-C
$$

where $N_{m}$ is the number of galaxies brighter than magnitude $m$. The investigations of Hubble ${ }^{1}$ and Mayall $^{2}$ give the distribution of extra-galactic nebulae to the 20.0 and 19.0 mag. respectively. From their results, values of $\log N_{m}$ were interpolated to the above limits corrected for red shift, and these points, when plotted on the present $\log N_{m}$ vs. $m$ curves, showed close agreement with the corresponding points for the brighter galaxies found in this investigation. The total absorptions found from the present data for the different regions ranged from 0.00 to 0.43 mag. and the average difference without regard 